\title{
Hand-position Oriented Humanoid Walking Motion Control System
}

\author{
Koichi Nishiwaki ${ }^{1}$, Yasutaka Fukumoto ${ }^{2}$, Satoshi Kagami ${ }^{1}$, Masayuki Inaba ${ }^{2}$, and \\ Hirochika Inoue ${ }^{1}$ \\ 1 Digital Human Research Center, AIST \\ 2-41-6, Aomi, Koto-ku, Tokyo, 135-0064, Japan \\ k.nishiwaki, s.kagami, h.inoue@aist.go.jp \\ 2 Dept. of Mechano-Informatics, The Univ. of Tokyo, \\ 7-3-1, Hongo, Bunkyo-ku, Tokyo, Japan \\ yasutaka,inaba@jsk.t.u-tokyo.ac.jp
}

\begin{abstract}
This paper addresses a method of constructing a control system that realizes desired absolute hand position trajectory while walking. Controlling hand position in the absolute coordinate system with short delay while walking is a important function for realizing such motions as visual feedback reaching while walking, walking using handrail, and opening doors while walking. Based on previously developed online walking control system, fast dynamically stable walking trajectory generation system that preserves designed hand trajectory in absolute coordinate system, and sensor feedback balance maintaining system that also preserves given hand position are developed. Then a torso transition planner that generates desired torso movement of every one step from the absolute hand trajectory is implemented. A hand position operation experiment with "3D mouse", a reaching experiment with visual feedback, and a guiding experiment using 6-axis force sensor at wrists are shown to demonstrate the performance of the system.
\end{abstract}

\section{Introduction}

Researches on biped walking of humanoid robots have been carried out actively, and robust walking control and walking that follows online commands are achieved (ex. [1-5]). We also constructed walking control system that follows online commands, such as, walking direction, step cycle, and upper body motion[6].

Usual humanoids have more degrees of freedom than it is required for walking, and expected to make use of many degrees of freedom efficiently for whole-body motion in human environment. The ability of using hands for other tasks while walking will be useful, as it realizes such motions as visual feedback reaching while walking, walking using handrail, pushing handcart, and opening doors while walking.

In order to realize those motions, we developed a online walking control system that accept desired hand position in absolute coordinate system with up to $1[\mathrm{kHz}]$ cycle. (It is same as motor servo control cycle.) Then we also developed torso transition planning system that generates desired torso movement of every one step from the hand movement information. Desired hand position trajectory will be the only input, and torso motion of every step and footprints are automatically generated from the input by the system. 


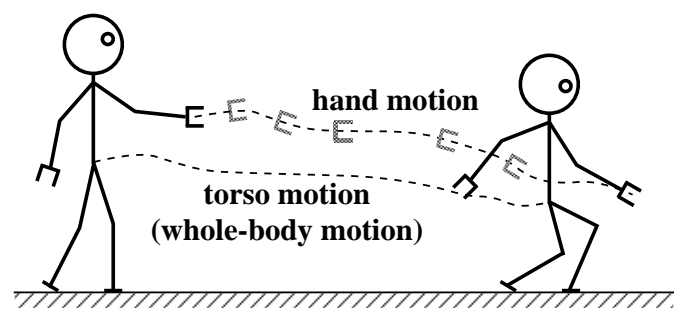

Fig. 1. Concept of hand-position oriented walking control.

\section{Hand-position Oriented Walking Control}

\subsection{Approach and Overview of System}

Our goal of this work is to realize a hand-position oriented humanoid walking control system that accepts the desired hand position trajectory in global coordinate system as input and generates and controls whole-body walking motion that satisfies the input (Fig. 1). Here obstacles around the robot are not considered, and the level plane is assumed for the ground in this work.

Almost humanoid walking is realized in 2 stages: dynamically stable motion trajectory generation using dynamics model, and sensor feedback modification of the trajectory. We have realized walking control system that follows online commands, such as, walking direction, step cycle, joint angle based arm motion. Dynamically stable motion trajectory generation is made online, and a planner that generates footprints from desired walking direction is implemented to realize the system[6].

We developed the hand-position oriented walking system based on this system. The overview of the system is shown in Fig. 2. Desired hand trajectory is passed to "Torso Transition / Footprint Planner". The planner generates footprints from the information every one step. Then "Walking Pattern Generator" makes dynamically stable walking trajectory that realizes given footprints and hand position trajectory. This trajectory generation is carried out just after the footprint planning in the same cycle. As the trajectory generation is carried out in simulation environment, generated trajectory is modified while execution using sensor information in order to maintain dynamic balance. This is the role of the "Sensor Feedback Balance Compensator". This compensator changes the horizontal torso position, but it does not change the hand position in absolute coordinate system from the given trajectory. It runs every $1[\mathrm{~ms}]$. If this compensator accept the desired hand position from the walking pattern generator, the update cycle of the desired hand position of whole system will be 1 step, and it is too large for sensor feedback and remote control system. Therefore the compensator is designed to accept the desired hand trajectory directly from the input. Therefore the update cycle of the desired hand position is $1[\mathrm{~ms}]$.

\subsection{Related Works}

Several works have been done focusing on a hand when dealing with whole-body motions. Inoue et al. proposed a method that changes body posture and makes a step in order to keep manipulability at hands[7]. Sian et al. have implemented a 


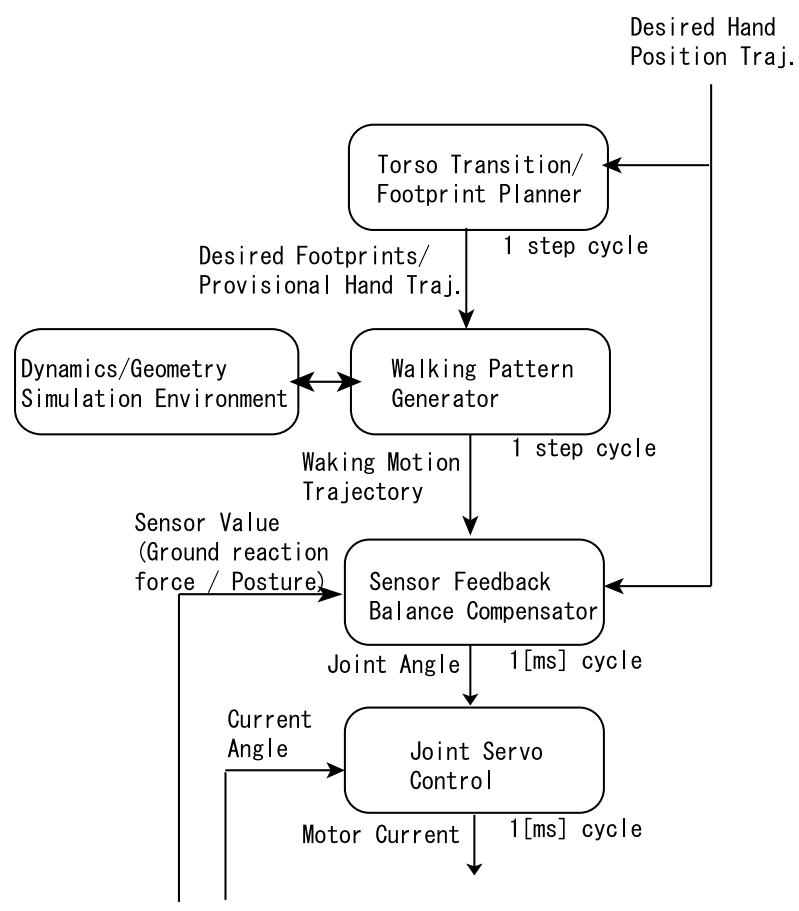

Fig. 2. Overview of the hand-position oriented walking control system.

"switching command based tele-operation system" which allows the operator to only manipulate specific points of the robot's body for generating stable whole-body motions[8]. Cooperative carrying of a panel with human and humanoid is presented by HRP2. Impedance control is employed for arm control and the hand position is not directly controlled in this demonstration[9].

We discuss how to realize desired hand trajectory while continuous walking is being executed in this paper.

\section{Walking Pattern Generation}

In this section, we will explain the "walking pattern generator". We adopted fast generation method of motion pattern that follows desired ZMP[10].

In general, ZMP trajectory can be analytically led from the robot motion trajectory, however leading robot trajectory that satisfies given ZMP trajectory analytically is difficult, since it has to solve non-linear, interfered $2^{\text {nd }}$ order differential equation with joint constraints. A fast generation method of walking trajectory that follows given ZMP trajectory by modifying horizontal torso trajectory from given initial trajectory is explained, in the following part. 


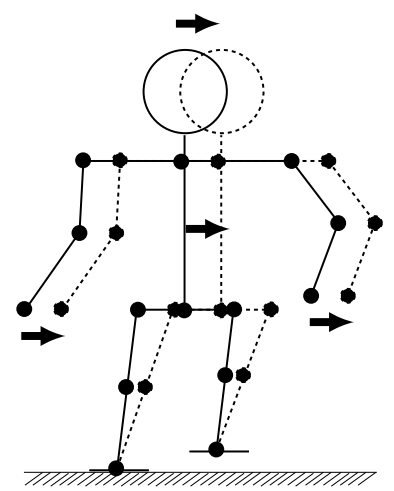

(a) Normal Generation

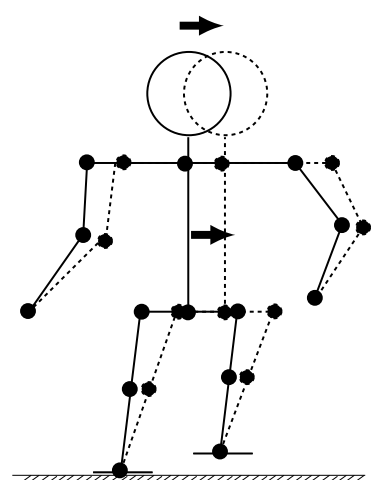

(b) Hand-fixed Generation

Fig. 3. Trajectory modification to obtain dynamically stable one.

\subsection{Linearlize and Non-Interferencize the Dynamics Equation about ZMP}

Let $i^{\text {th }}$ robot link position, mass, inertia tensor, angular velocity vector be $\mathbf{r}_{i}=$ $\left(x_{i}, y_{i}, z_{i}\right)^{\mathrm{T}}, m_{i}, \mathbf{I}_{i}, \omega_{i}$, and gravity be $g$. Set the coordinates as $x-y$ plane be the ground and as $z$-axis be negative gravity direction. Let ZMP be $\mathbf{P}=\left(x_{p}, y_{p}, 0\right)^{\mathrm{T}}$.

Then equation to lead ZMP from robot motion is described as follows (the same for $y_{p}$ );

$$
x_{p}=\frac{\sum m_{i} z_{i} \ddot{x}_{i}-\sum\left\{m_{i}\left(\ddot{z}_{i}+g\right) x_{i}+(0,1,0)^{\mathrm{T}} \mathbf{I}_{i} \dot{\boldsymbol{\omega}}_{i}\right\}}{-\sum m_{i}\left(\ddot{z}_{i}+g\right)} .
$$

Let robot trajectory be described as follows;

$$
\begin{aligned}
\mathbf{A}(t)= & \left(x_{1}(t), y_{1}(t), z_{1}(t), \theta_{1}(t), \phi_{1}(t), \psi_{1}(t),\right. \\
& \left.\cdots, x_{n}(t), y_{n}(t), z_{n}(t), \theta_{n}(t), \phi_{n}(t), \psi_{n}(t)\right) .
\end{aligned}
$$

ZMP trajectory $\mathbf{P}_{A}(t)=\left(x_{p_{a}}(t), y_{p_{a}}(t), 0\right)^{T}$ can be solved by Eq. (1). Now consider to generate trajectory that follows desired ZMP $\mathbf{P}_{A}^{*}(t)$ by only modifying $x_{i}(t), y_{i}(t)$ in $\mathbf{A}(t)$ to $x_{i}^{\prime}(t), y_{i}^{\prime}(t)$.

$$
x_{p}^{*}=\frac{\sum m_{i} z_{i} \ddot{x}_{i}^{\prime}-\sum\left\{m_{i}\left(\ddot{z}_{i}+g\right) x_{i}^{\prime}+(0,1,0)^{\mathrm{T}} \mathbf{I}_{i} \dot{\boldsymbol{\omega}}_{i}\right\}}{-\sum m_{i}\left(\ddot{z}_{i}+g\right)} .
$$

This problem is solving $x_{i}^{\prime}$ that satisfies Eq. (3) (the same for $y_{i}^{\prime}$ ). Eq. (1) - Eq. (3) gives the following equation;

$$
x_{p}^{e}=\frac{\sum m_{i} z_{i} \ddot{x}_{i}^{e}-\sum m_{i}\left(\ddot{z}_{i}+g\right) x_{i}^{e}}{-\sum m_{i}\left(\ddot{z}_{i}+g\right)} .
$$

Here, $x_{p}^{e}=x_{p}^{*}-x_{p_{a}}, x_{i}^{e}=x_{i}^{\prime}-x_{i}$. 


\subsection{Fixing feet and hands}

Since there is redundancy, consider $x_{i}^{e}=x^{e}$, that is, modifying the horizontal position of all the link in same distance. In reality, feet position can not be changed relative to the ground, however upper body can satisfy this modification. When modifying upper body position in horizontal plane, the position changes of leg links, which are led by inverse-kinematics, are approximately proportional to the upper body in horizontal plane and little for rotational and vertical component.

Therefore in the previous implementation we changed the upper body position in same distance, and leg link position is changed according to analytical (not redundant) inverse kinematics (Fig. 3(a)). For this work position of hands also should not be changed from initial trajectory. Therefore position of hands is also fixed, and position of arm links is decided by the Jacobian-based inverse kinematics (Fig. 3(b)).

\subsection{Numerical Solution of Differential Equation}

Set $x_{i}^{e}=x^{e}$ to Eq. (4), then following equation is obtained;

$$
-\frac{\sum m_{i} z_{i}}{\sum m_{i}\left(\ddot{z}_{i}+g\right)} \ddot{x}^{e}+x^{e}=x_{p}^{e} .
$$

In order to solve numerically time is discretized to $0, \cdots, t_{m}$ with time step $\Delta t$. Acceleration at each time $\ddot{x}^{e}\left(t_{i}\right)$ can be represented as follows;

$$
\ddot{x}^{e}\left(t_{i}\right)=\frac{x^{e}\left(t_{i+1}\right)-2 x^{e}\left(t_{i}\right)+x^{e}\left(t_{i-1}\right)}{\Delta t^{2}} .
$$

Then Eq. (5) can be expressed as trinomial equations. Setting boundary conditions as $x^{e}(0), x^{e}\left(t_{m}\right)=0, x^{e}(i)\left(i=1\right.$ to $\left.t_{m}-1\right)$ are obtained.

These values are the modification distance of every sampling time. In order to reduce the effect of the approximation, calculated trajectory is set to initial trajectory, and this procedure is repeated.

\section{Sensor Feedback Modification}

The original role of the sensor feedback modification layer is to handle the disturbance caused by modelling error of the robot and environment. Our robot falls down in several steps because of the difference between real world and modelled world without sensor feedback. We added a function of the updating hand posture according to the desired hand position input in absolute coordinate system. Low latency hand position control is achieved by this direct input.

This layer changes the horizontal position of the torso to maintain the balance. Position of feet is not changed from the given trajectory in absolute coordinate system, and position of hands is set to the input. Inverse kinematics is solved both for legs and arms.

Position of the robot in absolute coordinate system is obtained by dead reckoning. Therefore posture of the arms is calculated by desired absolute position of hands and torso position based on dead reckoning. Compensating the error caused by the dead reckoning is the role of outer loop, such as, visual feedback and remote operation. 

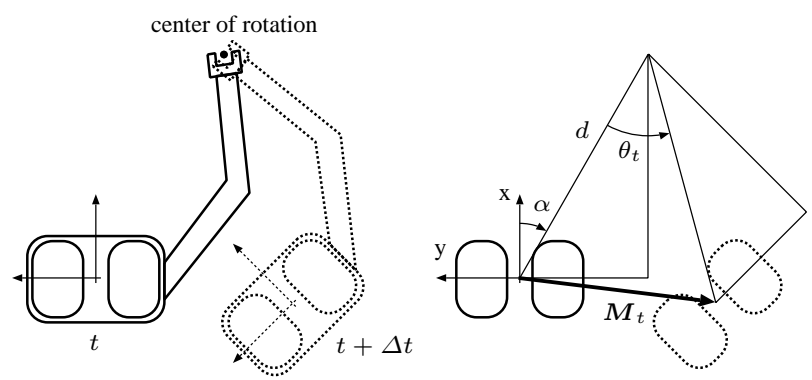

Fig. 4. Calculation of the torso trajectory.

\section{Inverse Kinematics of Arms}

We designed the robot arms to have 7 DOF for each, in order to extend the workspace. Pseudo jacobian is calculated to solve inverse kinematics of redundant system.

$$
d \boldsymbol{\theta}=\boldsymbol{J}^{\#} d \boldsymbol{x}+\left(\boldsymbol{I}-\boldsymbol{J}^{\#} \boldsymbol{J}\right) \boldsymbol{k}
$$

$k$ is chosen to make the joint which is the nearest to the angle limitation keep away from the limitation. However, solution falls to singularity point or joint angle limitation in some cases. Two emergency modes are prepared for such cases. One is to quit fixing hand position, and stop changing angles of the arm. This is used when the hand is in the air. The other is to cut off the power of motors in the arm. This option is used when the hand is grasping something connected to the environment.

\section{Planning of Torso Trajectory}

This system requires the inverse kinematics of the arm to be solved at all times. However, since it is hard to guarantee existence of the solutions of the inverse kinematics in advance, the following method is adopted. An initial arm posture is heuristically decided to be in the middle of the workspace and the robot tries to keep that configuration in average for the entire motion period.

The torso trajectory is determined so that the hand motion is canceled. A walking motion is generated to satisfy the desired torso motion vector $M_{t}$ (horizontal translation $M_{t, x}, M_{t, y}$ and rotation around vertical axis $\theta_{t}$ ) given at time $t$. Now suppose that the hand is rotating with respect to a point in space as shown in Fig. 4. In order for the torso to follow the hand by the time $t+\Delta t$, the torso motion vector $M_{t}$ should be

$$
\begin{aligned}
& M_{t, x}=2 d \sin \frac{\theta_{t}}{2} \sin \left(\alpha+\frac{\theta_{t}}{2}\right), \\
& M_{t, y}=-2 d \sin \frac{\theta_{t}}{2} \cos \left(\alpha+\frac{\theta_{t}}{2}\right),
\end{aligned}
$$



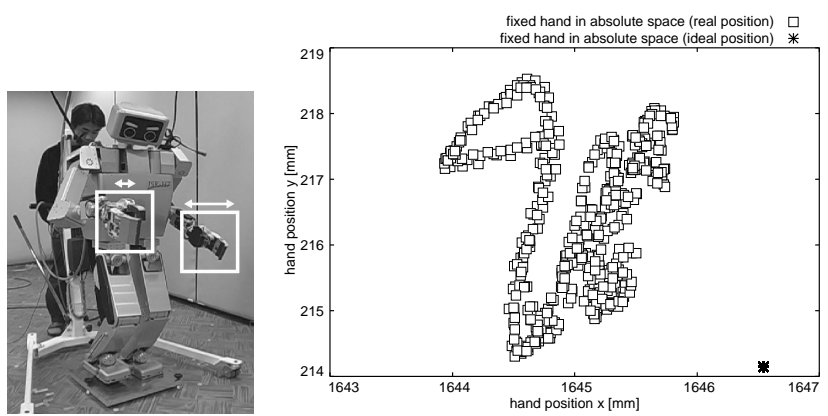

Fig. 5. Evaluation of the fixed hand while the robot is walking.

where $d$ denotes the initial distance between the hand and the torso frame, and $\alpha$ denotes the initial rotational angle of the origin of the hand frame with respect to the torso frame. The third element of the vector $M_{t}$ is equivalent to the rotational angle of the hand frame, which is $\theta_{t}$. The horizontal translation of the hand motion should be equal to that of the torso motion. So the horizontal translation of the hand motion is simply added to $M_{t, x}$ and $M_{t, y}$ in Eq. (8). Therefore, if the initial parameters $d$ and $\alpha$ are selected appropriately, the torso motion vector lets the body follow the hand motion. Then footprints are planned to realize the torso motion in average[11].

\section{Experiments and Evaluation}

The described functions are implemented, and online walking control system that realize desired hand trajectory in absolute coordinate system is constructed on humanoid "JSK-H7".

\subsection{Evaluation of the System}

For the first experiment, desired position of right hand is fixed to a point in order to evaluate the accuracy of the system. The oscillation of the hand was about 20 to 30 [mm]. The error is caused by the low rigidity of the links and joint servo error. In order to estimate the amount of joint servo error, direct kinematics is solved by using the current angles measured by encoders attached to the motors. Fig. 5 (right) shows the calculated result. Position of the right hand fluctuates in the maximum width of about $5[\mathrm{~mm}]$, and the average positions is not even near the goal hand position. Bias of the position is considered to be caused by gravity force and friction of the system. And the effect of low rigidity is relatively larger than the servo error.

\subsection{Remote Control Experiment}

Remote control system with 3D mouse (Magellan, 3Dconnexion) is constructed. The input from the mouse is converted to the desired velocity of a hand in the global coordinate system. The control point can be set to arbitral position in the hand link frame. As shown in Fig. 6, the robot successfully pushed the chair by two hands by setting the control point at the middle of the two hands, and controlling the both hand with same value. 

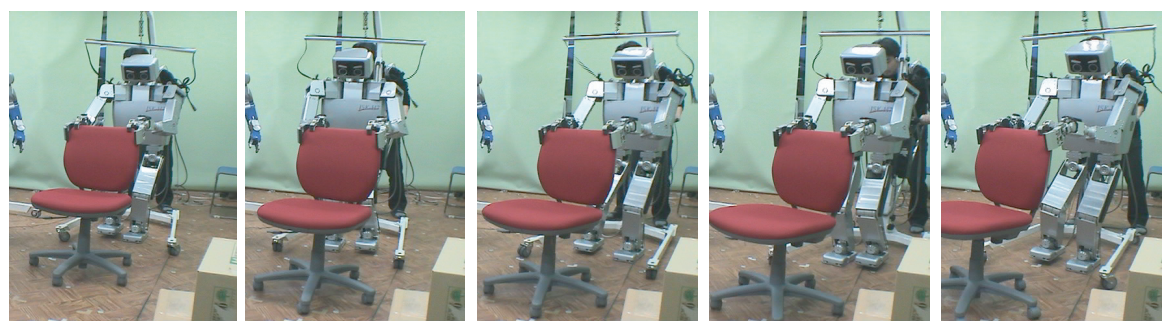

Fig. 6. Pushing a chair by remote control.

\subsection{Sensor Feedback Experiment}
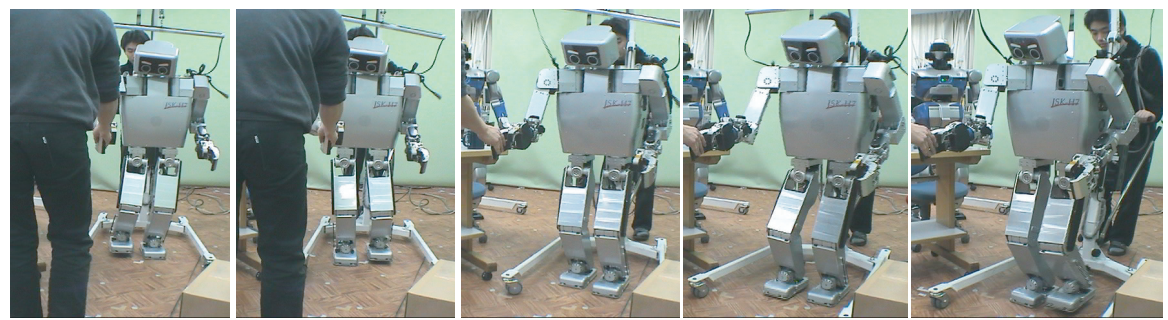

Fig. 7. Following a person using 6-axis force sensor at the wrist.

In Fig. 7, a person leads the robot by guiding its hand. 6-axis force information at the hand is calculated based on the output of the 6-axis force sensor at the wrist. The effect of gravity is approximately canceled by subtracting initial sensor output. Desired hand velocity is decided proportional to the 6-axis force. It is comfortable for the leading person that the dynamics compensation motion of the torso does not transmitted to the paerson. Furthermore, as the robot's hand follows the guiding person's hand in advance of the body, the person doesn't disturb the torso motion for stable walking.

\subsection{Visual Feedback Experiment}

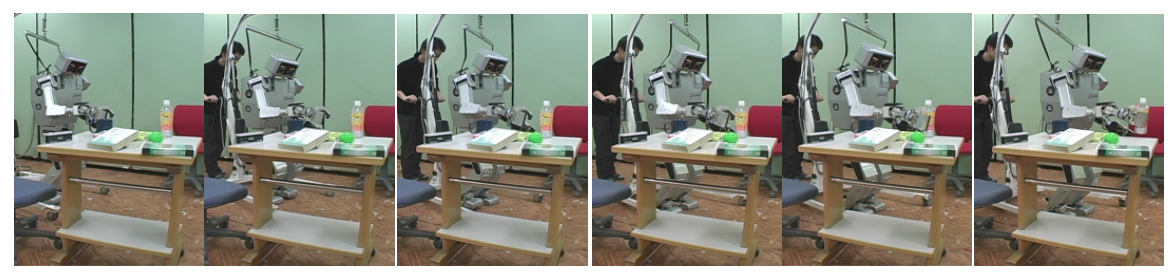

Fig. 8. Reaching while walking using visual feedback.

Reaching out for an object by watching it continuously becomes essential for a robust interaction between a hand and an environment. As the hand approaches the 
target object by visual servo, the robot grasps the object if it is settled in a certain distance from the hand.

Color markers are put on the hand and the target object. The 3D-position of the marker is calculated by means of trigonometrical measurement using the images of stereo camera on the head. Even though they have exactly the same color, they are distinguishable from each other using the arm posture. The 2D-position of the hand marker in the image coordinate system is estimated from the geometric information of the body. The robot judges whether the target object is in its hand or not by looking at a constant point near the visible hand marker. Because the robot observes the relative positions of the hand and the target, the gap between the actual and the ideal positions of the world frame does not have to be considered. The world frame moves as the robot's feet slip on the ground.

\section{Conclusion}

The method of constructing walking system that accepts trajectory of desired hand position and automatically generates and executes the walking motion is presented. The system is realized by designing and implementing three modules, torso transition planner, walking pattern generator, and sensor feedback balance compensator, that run cooperatively. We thought the system is indispensable for remote operation and sensor feedback tasks involved with hand position control. Some experiments of remote control and sensor feedback are shown that the system works well for such kinds of tasks.

For future work, we should develop more robust inverse kinematics to realize hand position, as most of the experimental failures arise from failing in solving inverse kinematics to realize the desired hand position during walking. We think using more DOFs than DOFs in the arm is important to make better inverse kinematics module. Giving up controlling some DOFs that is not important for a task also makes more robust inverse kinematics. Improving torso transition planner to use some prediction to decide the next step is another future work.

\section{References}

1. Kazuo Hirai. Current and future perspective of Honda humanoid robot. In Proc. of 1997 IEEE/RSJ International Conference on Intelligent Robots and Systems (IROS'97), pages 500-508, 1997.

2. Jin'ichi Yamaguchi, Sadatoshi Inoue, Daisuke Nishino, and Atsuo Takanishi. Development of a bipedal humanoid robot having antagonistic driven joints and three dof trunk. In Proc. of the 1998 IEEE/RSJ Int. Conf. on Intelligent Robots and Systems, pages 96-101, 1998.

3. Ken'ichirou Nagasaka, Masayuki Inaba, and Hirochika Inoue. Walking pattern generation for a humanoid robot based on optimal gradient method. In Proc. of 1999 IEEE Int. Conf. on Systems, Man, and Cybernetics, 1999.

4. Kazuhito Yokoi, Fumio Kanehiro, Kenji Kaneko, Kiyoshi Fujiwara, Shuji Kajita, and Hirohisa Hirukawa. A honda humanoid robot controlled by aist software. In Proc. of the IEEE-RAS International Conference on Humanoid Robots, pages 259-264, 2001. 
5. Yoshiharu Kaneshima, Yusuke Sugahara, Shumpei Ando, Motohiro Sato, Hun ok Lim, and Atsuo Takanishi. Quasi real-time motion pattern generation for bipedal humanoid robots. In Proceedings of 19th Annual Conference on Robotics Society of Japan, pages 987-988, 2001.

6. Koichi Nishiwaki, Satoshi Kagami, Yasuo Kuniyoshi, Masayuki Inaba, and Hirochika Inoue. Online generation of humanoid walking motion based on a fast generation method of motion pattern that follows desired zmp. In Proc. of IEEE/RSJ International Conference on Intelligent Robots and Systems (IROS'02), pages 2684-2689, 2002.

7. Kenji Inoue, Yusuke Nishihama, Tatsuo Arai, and Yasushi Mae. Mobile manipulation of humanoid robots -body and leg control for dual arm manipulation-. In Proc. of International Conference on Robotics and Automation (ICRA'O2), pages 2259-2264, 2002.

8. Neo Ee Sian, Kazuhito Yokoi, Shuuji Kajita, and Kazuo Tanie. Whole body teleoperation of a humanoid robot integrating operator's intention and robot's autonomy - an experimental verification -. In Proc. of IEEE/RSJ International Conference on Intelligent Robots and Systems (IROS'03), pages 1651-1656, 2003.

9. K. Yokoyama et al. Cooperative works by a human and humanoid (in japanese). In Proc. of The 21th Annual Conference of the Robotics Society of Japan, page 3A35, 2003.

10. S. Kagami, K. Nishwiaki, T. Kitagawa, T. Sugihara, M. Inaba, and H. Inoue. A fast generation method of a dynamically stable humanoid robot trajectory with enhanced zmp constraint. In Proc. of IEEE International Conference on Humanoid Robotics (Humanoid2000), 2000.

11. Koichi Nishiwaki, Satoshi Kagami, James J. Kuffner, Masayuki Inaba, and Hirochika Inoue. Online humanoid walking control system and a moving goal tracking experimant. In IEEE International Conference on Robotics and Automation (ICRA2003), pages 911916, 2003. 